\title{
Cohort Profile: Chinese Cervical Cancer Clinical Study
}

\author{
Xi-Ru Zhang ${ }^{1 \dagger}$, Zhi-Qiang $\mathrm{Li}^{2 \dagger}$, Li-Xin Sun ${ }^{3 \dagger}$, Ping $\mathrm{Liu}^{2,4}$, Zhi-Hao $\mathrm{Li}^{1}$, Peng-Fei $\mathrm{Li}^{2}$, \\ Hong-Wei Zhao ${ }^{3}$, Bi-Liang Chen ${ }^{5}$, Mei Ji ${ }^{6}$, Li Wang $^{7}$, Shan $\mathrm{Kang}^{8}$, Jing-He Lang ${ }^{9}$, Chen Mao ${ }^{1 *}$ \\ and Chun-Lin Chen ${ }^{2 *}$ on behalf of Chinese Cervical Cancer Clinical Study collaborative group ${ }^{\ddagger}$ \\ ${ }^{1}$ Department of Epidemiology, School of Public Health, Southern Medical University, Guangzhou, China, ${ }^{2}$ Department of \\ Obstetrics and Gynecology, Nanfang Hospital, Southern Medical University, Guangzhou, China, ${ }^{3}$ Department of Gynecologic \\ Oncology, Shanxi Provincial Cancer Hospital, Taiyuan, China, ${ }^{4}$ Department of Gynecology, Yanling Hospital of Southern \\ Medical University, Guangzhou, China, ${ }^{5}$ Department of Obstetrics and Gynecology, Xijing Hospital of Airforce Medical \\ University, Xi'an, China, ${ }^{6}$ Department of Obstetrics and Gynecology, The First Affiliated Hospital of Zhengzhou University, \\ Zhengzhou, China, ${ }^{7}$ Department of Gynecologic Oncology of Affiliated Cancer Hospital of Zhengzhou University, \\ Zhengzhou, China, ${ }^{8}$ Department of Gynecology, The Forth Hospital of Hebei Medical University, Shijiazhuang, China, \\ ${ }^{9}$ Department of Obstetrics and Gynecology, Peking Union Medical College Hospital, Peking Union Medical College, \\ Beijing, China
}

OPEN ACCESS

Edited by:

Pengpeng Qu,

Tianjin Central Hospital for Gynecology and Obstetrics, China

Reviewed by:

Aizhong Liu,

Central South University, China

Hui Wang,

Huazhong University of Science and

Technology, China

*Correspondence:

Chun-Lin Chen

ccl1@smu.edu.cn

Chen Mao

maochen9@smu.edu.cn

${ }^{\dagger}$ These authors have contributed equally to this work

FThe members of collaborative group of the Chinese Cervical Cancer Clinical

Study are listed in the Acknowledgments section

Specialty section:

This article was submitted to Gynecological Oncology, a section of the journal Frontiers in Oncology

Received: 02 April 2021 Accepted: 01 June 2021 Published: 18 June 2021

Citation:

Zhang $X-R$, Li Z-Q, Sun $L-X$, Liu P, Li Z-H, Li P-F, Zhao H-W, Chen B-L, Ji M, Wang L, Kang S, Lang J-H, Mao $C$ and Chen $C-L$ (2021) Cohort Profile: Chinese Cervical Cancer Clinical Study.

Front. Oncol. 11:690275. doi: 10.3389/fonc.2021.690275
Cervical cancer is the fourth most common cancer worldwide, but its incidence varies greatly in different countries. Regardless of incidence or mortality, the burden of cervical cancer in China accounts for approximately $18 \%$ of the global burden. The Chinese Cervical Cancer Clinical Study is a hospital-based multicenter open cohort. The major aims of this study include (i) to explore the associations of therapeutic strategies with complications as well as mid- and long-term clinical outcomes; (ii) to widely assess the factors which may have an influence on the prognosis of cervical cancer and then guide the treatment options, and to estimate prognosis using a prediction model for precise post-treatment care and follow-up; (iii) to develop a knowledge base of cervical clinical auxiliary diagnosis and prognosis prediction using artificial intelligence and machine learning approaches; and (iv) to roughly map the burden of cervical cancer in different districts and monitoring the trend in incidence of cervical cancer to potentially inform prevention and control strategies. Patients eligible for inclusion were those diagnosed with cervical cancer, whether during an outpatient visit or hospital admission, at 47 different types of medical institutions in 19 cities of 11 provinces across mainland China between 2004 and 2018. In a total, 63926 patients with cervical cancer were enrolled in the cohort. Since the project inception, a large number of standardized variables have been collected, including epidemiological characteristics, cervical cancer-related symptoms, physical examination results, laboratory testing results, imaging reports, tumor biomarkers, tumor staging, tumor characteristics, comorbidities, co-infections, treatment and short-term complications. Follow-up was performed at least once every 6 months within the first 5 years after receiving treatment and then annually thereafter. At present, we are developing a cervical cancer imaging database containing Dicom files with data of computed tomography/magnetic resonance imaging examination. Additionally, we are also collecting original pathological specimens of patients with cervical cancer. 
Potential collaborators are welcomed to contact the corresponding authors, and anyone can submit at least one specific study proposal describing the background, objectives and methods of the study.

Keywords: cervical cancer, cohort, therapy, prognosis, prediction

\section{HIGHLIGHTS}

- Starting in 2014, we developed the Chinese Cervical Cancer Clinical Study, a hospital-based open cohort, to assess outcomes of different management strategies on cervical cancers with specific clinical stages, and evaluate the influence of various prognostic factors on the oncological outcomes to guide treatment options, care, and follow-up.

- Up to now, this cohort has recruited 63926 patients with cervical cancer (mean [SD] age, 49.19 [10.44] years) from 47 general, cancer, as well as women's and children's hospitals distributed in 26 cities of 14 provinces across the mainland China.

- A total of 315 standardized variables were collected by reviewing the patients' medical records, which covered almost all information on epidemical characteristics, clinical testing results, examinations clinical diagnoses, treatments and prognosis at baseline. Follow-up was conducted at certain time intervals.

- We are building a cervical cancer imaging database to collect the Dicom file data of computed tomography and/or magnetic resonance imaging examinations. Additionally, we are collecting original pathological samples of patients with cervical cancer. Based on those, we might conduct extensive researches on imaging omics and pathology omics of cervical cancer using artificial intelligence, machine learning and advanced biological technology.

\section{WHY WAS THE COHORT SET UP?}

Cervical cancer is a major public health problem. It is the fourth most common cancer type in terms of incidence and mortality in women worldwide. In 2018, an estimated 570000 cases of cervical cancer and 311000 deaths caused by cervical cancer have been recorded (1-5). The incidence and mortality rates of cervical cancer varied widely among countries (1). With the highest number of cases (106 430) and the second-highest estimated number of deaths (47 739), China accounts for approximately $18 \%$ of the global cervical cancer burden (6).

The elimination of cervical cancer as a public health issue is considered a priority under the WHO 13th General Programmer of Work of the World Health Organization (7-9). Despite the wide availability of screening and improved therapeutic practices, the 5-year overall survival (OS) of cervical cancer remains at only $60-70 \%$ in high-income countries and it is much lower in middle- and low-income countries (10). Therefore, more reasonable prevention and control strategies must be developed. And more effective diagnosis and treatment strategies must be applied in clinical practice. These strategies are associated with a shorter potential years of life lost and a fewer disability-adjusted life year lost due to cervical cancer (11).

The effect of different management on patients with specific clinical stages of cervical cancer remains controversial (12-15). Prognostic factors, including tumor characteristics, medical condition, sociodemographic characteristics, lifestyle behaviors, biomarkers, diagnosis, treatment, and care, are suggested for further detailed exploration (16-22). This may provide a potential way to reduce residual disease after treatment, as well as minimize recurrence, decrease the complications, and improve survival. Furthermore, the recent advances in medical information technology and computer technology play an important role in the realization of auxiliary diagnosis and digital healthcare of cervical cancer $(23,24)$. These developments provide an optimism outlook for remarkable progress in the diagnosis, treatment, and prediction of the prognosis of cervical cancer.

The Chinese Cervical Cancer Clinical (Four-C) Study was created in 2014 with the aim of collecting clinical and prognostic information on patients diagnosed with cervical cancer in mainland China since 2004. Its research objectives currently focus on four main themes: (i) to explore the associations of therapeutic strategies with complications as well as mid- and long-term clinical outcomes, including comparative effectiveness research based on marginal structural models or propensity scores (25-27); (ii) to widely evaluate the prognostic factors of cervical cancer (such as late access to care and the influence of nutritional status) and then guide treatment as well as care options, and to precisely predict the prognosis of patients so as to develop much more effective program of personalized followup and intervention $(21,22)$; (iii) to utilize artificial intelligence (AI) and machine learning (ML) approaches for multimodal data aggregation and multifactorial examination in order to develop a knowledge base of cervical clinical auxiliary diagnosis and prognostic prediction (28-30). What' more, as the Four-C Study relatively represents the occurrence of cervical cancer across mainland China in terms of age, geographical origin, year of diagnosis, clinical stage, gross type, and histological type, it can also serve to map the burden of cervical cancer in different districts and monitor trends in incidence of cervical cancer, which could potentially inform prevention and control strategies (31).

\section{WHO IS IN THE COHORT?}

The Four-C Study was set up by two phases. The inclusion criteria for the participants were as follows: subjects who were 
outpatients or inpatients of participating centers of the Four- $\mathrm{C}$ Study; subjects who have a pathology report of cervical biopsy, which is the gold standard for cervical cancer diagnosis, issued by at least two experienced doctors in a Grade III Level A hospital. In the first phase initiated in 2014, the hospital-based open cohort recruited 46205 patients diagnosed with cervical cancer between 2004 and 2016 at 37 hospitals distributed in 19 cities of 11 provinces across mainland China. The second phase was launched in 2019. Three of the original 37 hospitals (Guizhou Provincial People's Hospital, Shanxi Provincial Cancer Hospital, and The Yuncheng Central Hospital) continuously participated in the second phase. Additionally, other 10 hospitals collected epidemiological and clinical data of 17721 patients who was diagnosed with cervical cancer between 2004 and 2018. In a total, 63926 patients with cervical cancer from 47 general hospitals, cancer hospitals, as well as women's and children's hospitals distributed in 26 cities of 14 provinces across the mainland China were enrolled in the large-scale open subject cohort (Figure 1). The geographical distribution of all the participants is shown in Figure 2.

The documentation of patient information included epidemical characteristics, clinical testing results, examinations, diagnoses, treatments, care and prognosis. Further details on the project are available at the International Clinical Trails Registry Platform (http://apps.who.int/trialsearch/, ChiCTR1800017778). Approval was obtained from the institutional ethics committee of Nanfang Hospital (NFEC-2017-135) (32, 33). Only the information on the medical practice of each patient was collected so that individual patients could not be identified. The ethics committee exempted informed consent. Of note, every patient is assigned a unique number to match the clinical/epidemiological information obtained from medical records with original pathology specimens or image data, which can be used to validate a diagnosis or provide additional information for subsequent specific research projects.

\section{WHAT HAS BEEN MEASURED AND COLLECTED?}

At baseline, the Four-C Study collected 315 epidemiological and clinical variables by returning to the patients' medical records, which covered almost all information on clinical diagnosis, treatment, care and short-term outcome of cervical cancer, including sociodemographic characteristics, menstrual history and reproductive history, type of medical institution, cervical cancer-related symptoms, physical examination, laboratory testing, imaging report, tumor biomarkers, tumor staging, tumor characteristics, comorbidities, co-infections, management of patients and short-term complications after treatments. Table 1 shows a broad overview of the information collected on each inclusion participant. Further detailed information on management of patients, such as surgical approaches, surgical procedures, other surgical-related information, and the complete protocols radiotherapy or chemotherapy, were also documented, which was summarized in Table 2. The tumor staging was defined by the International Federation of Gynecology and Obstetrics (FIGO) staging system in 2009 and 2018.

The distribution of baseline characteristics was displayed as the number (percentage [\%]) for categorical variables and mean (standard deviation [SD]) for continuous variables (Table 3). Of the 63926 patients (mean [SD] age, 49.19 [10.44] years), more than one-half (58.9\%) lived in rural areas, approximately $70 \%$ lived in a second-tier city and $95.4 \%$ were married. When giving birth, vaginal delivery (79.6\%) was the most common delivery method. Patients were most likely to receive treatment in a general hospital (31 997 cases, 50.1\%), followed by a cancer center $(29893,46.8 \%)$. There are $49852(78.0 \%)$ patients with FIGO stage I-II, 5663 patients (8.9\%) with FIGO stage III and 689 patients $(1.1 \%)$ with FIGO stage VI. The gross tumor type was mainly exogenous (41.7\%), and the pathological tumor type

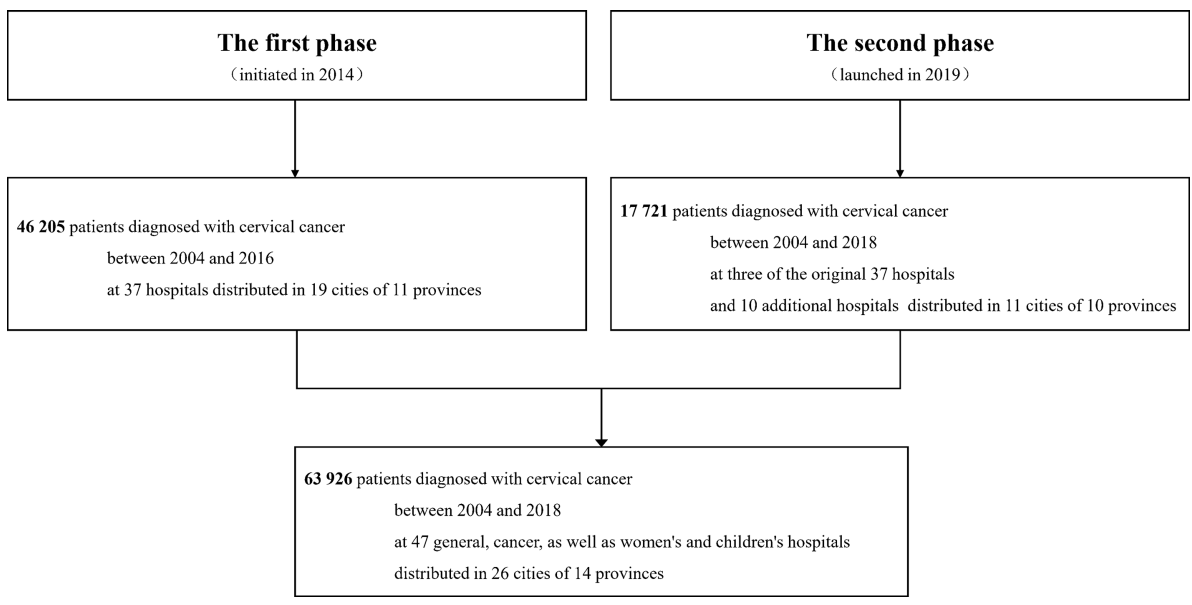

FIGURE 1 | Flowchart of participant enrolment. 


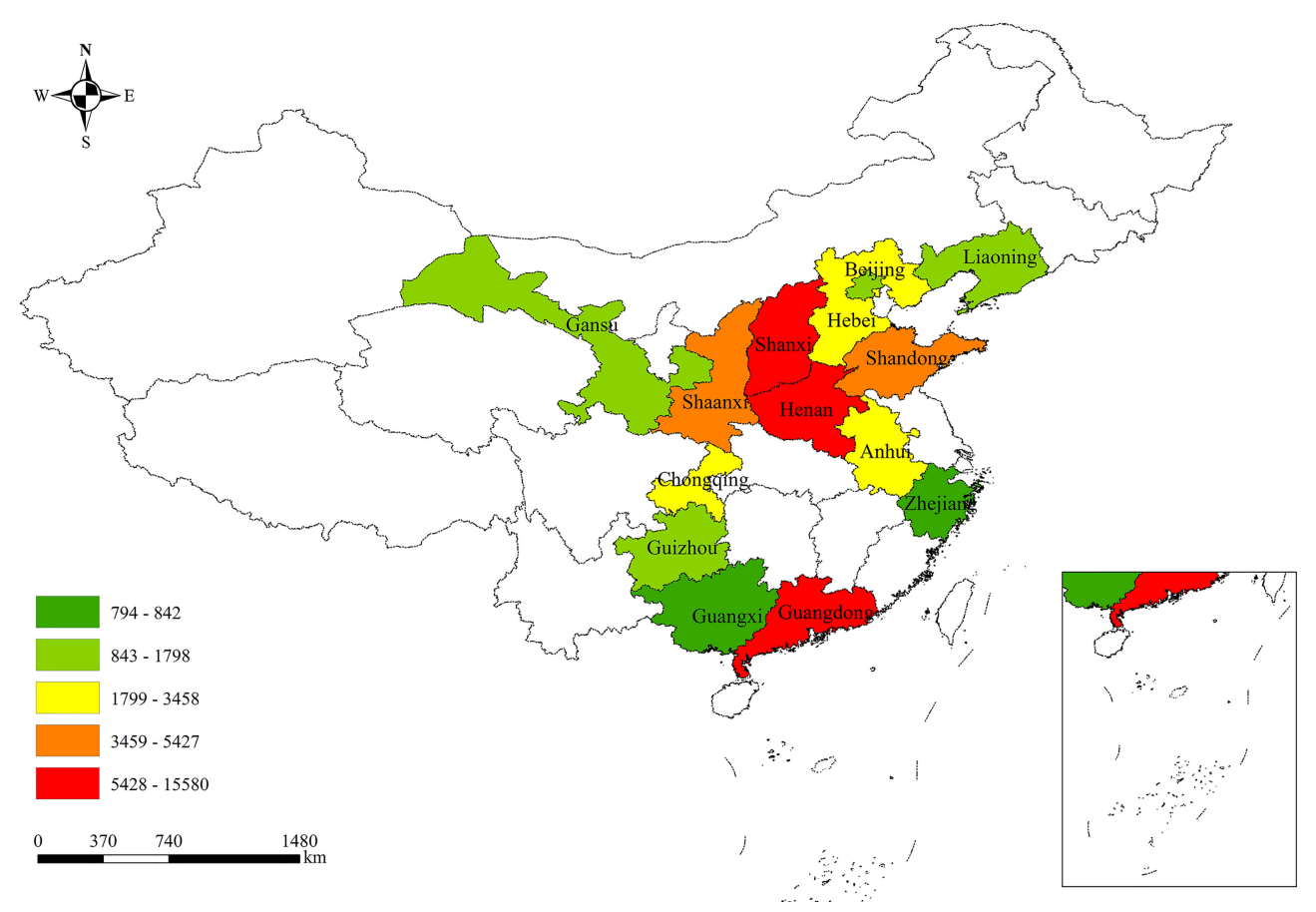

FIGURE 2 | The province distribution of the study participants.

was generally squamous cell carcinoma $(87.8 \%)$ and adenocarcinoma (6.9\%). Of the 48962 patients who underwent surgery, abdominal hysterectomy was reported for 28482 cases (58.2\%), laparoscopic surgery for 15499 (31.7\%), robot-assisted hysterectomy for 2176 cases (4.4\%) and vaginal hysterectomy for $628(1.3 \%)$.

\section{HOW OFTEN HAVE THEY BEEN FOLLOWED UP?}

Follow-up was conducted by well-trained nursers, research assistants and postgraduates in Gynecology and Obstetrics through telephone conversations with the patient or one of her family members, or via the assessment of patient electronic medical notes (both at each outpatient visit or hospital admission) at certain time intervals defined by the follow-up strategy. The follow-up interval is every 3 to 4 months within the first 2 years, every 6 months for the next 3 years, and then annually thereafter or until year 10 at the discretion of the treating physician. Bedsides, detailed follow-up information of participants is require to be collected immediately if a special and meaningful clinical manifestation is identified or a significant change in tumor-related biological markers is noted during an outpatient visit for review after treatment.

Standardized variables were collected to evaluate the residual disease, residual tumor, surveillance, survival, and other information (Table 1). These included the time of follow-up, middle- and long-term complications, treatment strategies for complications, recurrence of cervical cancer and the time of recurrence, site of recurrence and treatment after recurrence, drug use, outpatient, hospitalization and death information.

We take some measures to minimize the loss of follow-up. Firstly, all hospitals have promised to strictly abide by the followup strategy. Upon discharge or departure from the clinic, each participant was notified in detail the time interval for reexamination and/or follow-up. The doctors in charge should maintain long-term communication with each patient to improve their compliance. Additionally, patients who move or seek care elsewhere were also traced, and information of those who died was collected through telephone conversations with one of her close family members.

\section{QUALITY ASSURANCE AND CONTROL}

First of all, after full discussion, preextraction and revision, a concise and standardized case report form (CRF) and a brief guideline for performing data extraction from the medical records were developed. Baseline information was abstracted from two systems: an electronic medical record (EMR) system and a conventional paper medical record kept in the hospital medical documents room, which ensured the integrity of data. Data were read and entered into electronic CRF by two trained gynecologists, nurses or postgraduates in obstetrics and gynecology, respectively. Subsequently, we checked the 
TABLE 1 | Epidemiological and clinical data in the Chinese Cervical Cancer Clinical Study.

\begin{tabular}{|c|c|}
\hline Components & Measurements \\
\hline \multicolumn{2}{|l|}{ Baseline data } \\
\hline Sociodemographic characteristics & $\begin{array}{l}\text { date of birth, age at first diagnosis, ethnicity, region, Province, City, city scale, education level, occupation, residence, marital } \\
\text { status, age of marriage, sexual life and family history of cervical cancer }\end{array}$ \\
\hline Menstrual history and reproductive & age of menarche, pregnancy history, age of childbearing, parity, delivery way and hormone replacement therapy history \\
\hline \multicolumn{2}{|l|}{ history } \\
\hline Type of medical institution & general hospital, cancer center, women and children's center \\
\hline Related symptoms & $\begin{array}{l}\text { anemia, leukorrhagia, irregular vaginal bleeding, contact bleeding, a foul-smelling watery or sometimes bloody vaginal } \\
\text { discharge, lower extremity edema, fever, oliguria or osphyalgia }\end{array}$ \\
\hline Physical examination & $\begin{array}{l}\text { bimanual pelvic examination, colposcopy, biopsy, height, weight, resting blood pressure, temperature, pulse, heart rate, the } \\
\text { administration of 12-lead electrocardiography (ECG), auscultation heart and lung, hearing acuity, regular examinations } \\
\text { of otorhinolaryngology, the heart and blood vessels examination, respiratory system examination, nervous system examination } \\
\text { and abdominal viscera examination, limbs and joints movements, liver function }\end{array}$ \\
\hline Laboratory testing & thinprep cytologic test (TCT), human papillomavirus testing (HPV testing), pathology report \\
\hline Imaging report & $\begin{array}{l}\text { computed tomography (CT), magnetic resonance imaging (MRI), endovaginal/transrectal ultrasound (US) and/or positron } \\
\text { emission tomography-computed tomography (PET-CT) }\end{array}$ \\
\hline Tumor biomarkers & $\begin{array}{l}\text { squamous cell carcinoma antigen (SCC-Ag), carcinoembryonic antigen (CEA), Human epididymis protein } 4 \text { (HE4), } \\
\text { carbohydrate antigen-153 (CA-153), Carbohydrate antigen199 (CA-199), carbohydrate antigen-125 (CA-125), tissue } \\
\text { polypeptide antigen (TPA), tumor necrosis factor alpha (TNF- } \alpha \text { ) and interleukin- } 6 \text { (IL-6) }\end{array}$ \\
\hline Tumor characteristics & $\begin{array}{l}\text { tumor size, tumor location, pathological tumor type, gross type, tumor volume, a maximum depth of cervical stromal invasion, } \\
\text { a minimum thickness of uninvolved cervical stroma, extracervical tumor extension and LN involvement (number, size, location), } \\
\text { clinically visible lesion, presence or absence of lymph vascular space involvement (LVSI), presence or absence of distant } \\
\text { metastases, TNM classification }\end{array}$ \\
\hline FIGO stage & Using the International Federation of Gynecology and Obstetrics (FIGO) staging system in 2009 and 2018 \\
\hline Comorbidities & malignancies, hypertension, CVD, diabetes, kidney disease, pulmonary disease, gallbladder disorders \\
\hline Medical and medication history & $\begin{array}{l}\text { Surgical, drugs taken for diabetes, hypertension, CVD, kidney disease, respiratory diseases and digestive disease and } \\
\text { immunosuppressive drug }\end{array}$ \\
\hline Co-infection & hepatitis virus, herpes simplex virus (HSV), treponema pallidum, human immunodeficiency virus (HIV) or other virus \\
\hline Management of patients & $\begin{array}{l}\text { surgical treatment, primary radiotherapy, primary chemoradiotherapy, adjuvant radiotherapy, adjuvant chemo(radio)therapy, } \\
\text { neoadjuvant chemotherapy, Neoadjuvant brachytherapy, hospital stay }\end{array}$ \\
\hline Short-term complications & vascular injury, bladder injury, ureteral injury, bowel injury, stomach injury obturator nerve injury \\
\hline \multicolumn{2}{|l|}{ Follow-up data } \\
\hline Middle- and long-term complications & $\begin{array}{l}\text { rectovaginal fistula, vesicovaginal fistula, ureterovaginal fistula, ureteral fistula, lymphedema, bowel obstruction, pelvic } \\
\text { hematoma, venous thromboembolism, hemorrhage, chylous leakage, radiation proctitis, vaginitis, vulvitis, and bone marrow } \\
\text { suppression }\end{array}$ \\
\hline Management & Reexamination, treatment strategies for complications, care, hospitalization and medication \\
\hline Recurrence & time of recurrence, location of recurrence and treatment after recurrence \\
\hline Drug use & Drugs taken for diabetes, hypertension, CVD, kidney disease, respiratory diseases or digestive disease \\
\hline Death information & time of death, cause of death \\
\hline
\end{tabular}

LN, lymph node; CVD, cardiovascular disease.

TABLE 2 | Detailed information on treatment.

\section{Components}

Measurements

Surgical treatment Surgical approaches abdominal, vaginal, laparoscopic or robot-assisted

Surgical procedures cone/loop resection, resection margins, presence or absence of positive resection margins, trachelectomy, type of hysterectomy, nerve-sparing radical surgery, presence or absence of ovaries and fallopian tubes, lymphadenectomy, LN dissection, presence or absence of vaginal cuff, and presence or absence of parametria

Other information preoperative care, preoperative workup, pretreatment surgical, anesthesia, surgical margins, surgical interventions, blood transfusion, intraoperative blood loss, operation period, postoperative care, postoperative complications, postoperative recurrence, the time of postoperative first exhaust and defecation, the time of postoperative catheter removal, postoperative residual urine, residual disease, residual tumor or reoperation,

Radiotherapy type of radiation source, radiation approach, area exposed, site exposed, unit dose, total dose, total number of segments and total treatment time as well as the effectiveness of radiotherapy

Chemotherapy drug, dose, course, interval and route

LN, lymph node.

consistency of data for the same patient entered by different researchers and tried our efforts to correct any questionable data. Meanwhile, the information in the database was randomly examined by researchers designated by the study group. In this way, the accuracy and completeness of all the input information were guaranteed, even though it required significant labor and time costs. Finally, editing of the database was locked to prevent the modification or destruction of the determined data. 
TABLE 3 | Selected characteristics of participants in the Chinese Cervical Cancer Clinical Study.

\begin{tabular}{|c|c|c|c|c|}
\hline \multirow[t]{2}{*}{ Characteristics } & \multirow[t]{2}{*}{ Overall ( $N=63$ 926) } & \multirow[t]{2}{*}{ The First stage ( $N=46$ 205) } & \multicolumn{2}{|c|}{ The second stage $(\mathrm{N}=17 \mathrm{721})$} \\
\hline & & & RRH Data (N = 2790) & General data $(\mathrm{N}=14931)$ \\
\hline Age at first diagnosis, years & $49.19(10.44)$ & $49.34(10.55)$ & $48.12(10.05)$ & $48.93(10.12)$ \\
\hline \multicolumn{5}{|l|}{ Age at first diagnosis, years } \\
\hline$\leq 45$ & 24413 (38.19) & 17586 (38.06) & 1117 (40.04) & $5710(38.25)$ \\
\hline $46-69$ & 37060 (57.97) & $26642(57.66)$ & $1636(58.64)$ & $8782(58.82)$ \\
\hline$\geq 70$ & $2190(3.43)$ & 1768 (3.83) & $37(1.33)$ & $385(2.58)$ \\
\hline Missing & $263(0.41)$ & $209(0.45)$ & 0 & $54(0.36)$ \\
\hline \multicolumn{5}{|l|}{ Year of diagnosis } \\
\hline 2004-2006 & $4662(7.3)$ & $3891(8.4)$ & $25(0.9)$ & $746(5.0)$ \\
\hline $2007-2010$ & $13685(21.4)$ & $11585(25.1)$ & $62(2.2)$ & 2038 (13.7) \\
\hline 2011-2014 & $24972(39.1)$ & $20886(45.2)$ & $650(23.3)$ & $3436(23.0)$ \\
\hline $2015-2018$ & 20599 (32.2) & 9843 (21.3) & $2053(73.6)$ & 8703 (58.3) \\
\hline \multicolumn{5}{|l|}{ Marital status } \\
\hline Married & 61002 (95.43) & 43757 (94.70) & 2667 (95.59) & 14578 (97.64) \\
\hline Unmarried & $306(0.48)$ & $226(0.49)$ & $23(0.82)$ & $57(0.38)$ \\
\hline Divorced & $684(1.07)$ & $512(1.11)$ & $50(1.79)$ & $122(0.82)$ \\
\hline Widowed & $671(1.05)$ & $529(1.14)$ & $39(1.40)$ & $103(0.60)$ \\
\hline Remarried & $231(0.36)$ & $200(0.43)$ & $11(0.39)$ & $20(0.13)$ \\
\hline Unknown & $1032(1.61)$ & $981(2.12)$ & 0 & $51(0.34)$ \\
\hline \multicolumn{5}{|l|}{ Residence } \\
\hline Rural & $37626(58.86)$ & $26791(57.98)$ & $1422(51.00)$ & $9413(63.00)$ \\
\hline Urban & $18419(28.81)$ & 12782 (27.66) & $1027(36.80)$ & $4610(30.90)$ \\
\hline Unknow & 7881 (12.33) & $6632(14.35)$ & 341 (12.20) & $908(6.10)$ \\
\hline \multicolumn{5}{|l|}{ Region } \\
\hline North & $19653(30.7)$ & 18410 (39.8) & $400(14.3)$ & $843(5.60)$ \\
\hline South & $10889(17.0)$ & 10633 (23.0) & $256(9.2)$ & 0 \\
\hline Central & $11571(18.1)$ & 7092 (15.3) & $306(11.0)$ & 4173 (27.9) \\
\hline East & 7305 (11.4) & $4563(9.9)$ & 0 & $2742(18.4)$ \\
\hline Southwest & $5865(9.2)$ & 5507 (11.9) & 0 & $358(2.4)$ \\
\hline Northwest & 6499 (10.2) & 0 & $1828(65.5)$ & 4671 (31.3) \\
\hline Northeast & $2144(3.4)$ & 0 & 0 & 2144 (14.4) \\
\hline \multicolumn{5}{|l|}{ City scale } \\
\hline First-tier & 8948 (14.0) & 8548 (18.5) & $400(14.3)$ & 0 \\
\hline Second-tier & 44929 (70.3) & 29006 (62.8) & 2310 (82.8) & $13613(91.2)$ \\
\hline Third-tier and below & $10049(15.7)$ & 8651 (18.7) & $80(2.90)$ & $1318(8.8)$ \\
\hline \multicolumn{5}{|l|}{ Institution type } \\
\hline General hospital & $31997(50.1)$ & $16000(34.6)$ & $2534(90.8)$ & $13463(90.2)$ \\
\hline Cancer center & $29893(46.8)$ & 28425 (61.5) & 0 & $1468(9.8)$ \\
\hline Women and children center & $2036(3.2)$ & $1780(3.9)$ & $256(9.2)$ & 0 \\
\hline \multicolumn{5}{|l|}{ Delivery types } \\
\hline No delivery & $624(0.98)$ & $529(1.14)$ & $38(1.36)$ & $57(0.38)$ \\
\hline Vaginal delivery & $50864(79.57)$ & 35449 (76.72) & 2332 (83.58) & $13083(87.62)$ \\
\hline Cesarean delivery & 2669 (4.18) & 1643 (3.56) & $229(8.21)$ & $797(5.34)$ \\
\hline Vaginal and cesarean delivery & $822(1.29)$ & $524(1.13)$ & $71(2.54)$ & $227(1.52)$ \\
\hline Unknown & $8947(14.00)$ & $8060(17.44)$ & $120(4.30)$ & $767(5.14)$ \\
\hline \multicolumn{5}{|l|}{ FIGO stage (2009) } \\
\hline $\mid \mathrm{A} 1$ & 2496 (3.90) & $1657(1.07)$ & $105(3.76)$ & $734(4.92)$ \\
\hline IA2 & $714(1.12)$ & $496(27.83)$ & $42(1.51)$ & $176(1.18)$ \\
\hline IB1 & $18036(28.21)$ & $12858(7.92)$ & $1182(42.37)$ & $3996(26.76)$ \\
\hline IB2 & $4716(7.38)$ & $3660(1.54)$ & $231(8.28)$ & 825 (5.53) \\
\hline IA & $1056(1.65)$ & $713(1.26)$ & $36(1.28)$ & $307(2.06)$ \\
\hline $\mathrm{IB}$ & $928(1.45)$ & $581(11.86)$ & $30(1.08)$ & $317(2.12)$ \\
\hline$\| \mathrm{A} 1$ & 7702 (12.05) & $5479(6.40)$ & $471(16.88)$ & $1752(11.73)$ \\
\hline$\| \mathrm{A} 2$ & $3901(6.10)$ & $2956(2.32)$ & $211(7.56)$ & $734(4.92)$ \\
\hline$\| A$ & 1455 (2.28) & $1071(12.40)$ & $26(0.93)$ & $358(2.40)$ \\
\hline IIB & 8542 (13.36) & 5729 (1.25) & 302 (10.82) & $2511(16.82)$ \\
\hline IIIA & 772 (1.21) & $576(8.30)$ & 17 (0.61) & 179 (1.20) \\
\hline IIIB & 4680 (7.32) & $3833(0.28)$ & $25(0.90)$ & $822(5.51)$ \\
\hline 1 & 177 (0.28) & $129(0.23)$ & 0 & 48 (0.32) \\
\hline$\|$ & $129(0.20)$ & $108(0.41)$ & $3(0.11)$ & $18(0.12)$ \\
\hline III & $211(0.33)$ & $188(0.25)$ & $1(0.04)$ & $22(0.15)$ \\
\hline IVA & $145(0.23)$ & $115(0.49)$ & $2(0.07)$ & $28(0.19)$ \\
\hline
\end{tabular}


TABLE 3 | Continued

\begin{tabular}{|c|c|c|c|c|}
\hline \multirow[t]{2}{*}{ Characteristics } & \multirow[t]{2}{*}{ Overall (N = 63 926) } & \multirow[t]{2}{*}{ The First stage ( $N=46$ 205) } & \multicolumn{2}{|c|}{ The second stage $(\mathrm{N}=17 \mathrm{721})$} \\
\hline & & & RRH Data (N = 2790) & General data ( $N=14931)$ \\
\hline IVB & $241(0.38)$ & $228(0.47)$ & $1(0.04)$ & $12(1.08)$ \\
\hline IV & $303(0.47)$ & $217(1.38)$ & 0 & $86(0.58)$ \\
\hline $\mathrm{CIN}$ & $987(1.54)$ & 637 (10.77) & $7(0.25)$ & $343(2.30)$ \\
\hline Unknown & 6735 (10.54) & $4974(10.54)$ & $98(3.51)$ & 1663 (11.14) \\
\hline \multicolumn{5}{|l|}{ Gross type } \\
\hline Erosion & 809 (1.3) & $809(1.8)$ & 0 & 0 \\
\hline Exophytic & $26630(41.7)$ & $19154(41.5)$ & 1479 (53.0) & 5997 (40.2) \\
\hline Endophytic & $5951(9.3)$ & 4993 (10.8) & $98(3.5)$ & $860(5.8)$ \\
\hline ulcerative & $7487(11.7)$ & $6686(14.5)$ & $115(4.1)$ & $686(4.6)$ \\
\hline Cervical canal & $776(1.2)$ & $551(1.2)$ & $109(3.9)$ & $116(0.8)$ \\
\hline After conization & 2047 (3.2) & $1276(2.8)$ & $111(4.0)$ & $660(4.4)$ \\
\hline No found & $16347(25.6)$ & $11130(24.1)$ & $785(28.1)$ & 4432 (29.7) \\
\hline Unknown & $3879(6.1)$ & $1606(1.8)$ & $93(3.3)$ & $2180(14.6)$ \\
\hline \multicolumn{5}{|l|}{ pathological tumor type } \\
\hline Squamous cell carcinoma & $56141(87.8)$ & 40612 (87.9) & 2455 (88.0) & $13074(87.6)$ \\
\hline Adenocarcinoma & $4422(6.9)$ & $3037(6.6)$ & $243(8.7)$ & $1142(7.8)$ \\
\hline Adenosquamous carcinoma & $992(1.6)$ & $757(1.6)$ & $43(1.5)$ & $192(1.3)$ \\
\hline Clear cell carcinoma & $117(0.2)$ & $90(0.2)$ & $4(0.1)$ & $23(0.2)$ \\
\hline Small cell neuroendocrine carcinoma & $372(0.6)$ & $279(0.6)$ & $1(0.0)$ & $92(0.6)$ \\
\hline Other subtypes & $518(0.8)$ & $376(0.8)$ & $29(1.0)$ & $113(0.8)$ \\
\hline Unknown & $1364(2.1)$ & $1054(2.3)$ & $15(0.5)$ & $295(2.0)$ \\
\hline
\end{tabular}

FIGO, the International Federation of Gynecology and Obstetrics; CIN, cervical intraepithelial neoplasia.

Notably, all data were backed up at every stage of database development to prevent accidental loss of recorded data.

\section{WHAT HAS IT FOUND? KEY FINDINGS AND PUBLICATIONS}

Although laparoscopic surgery and robot-assisted hysterectomy for cervical cancer have significantly increased since 2004, little is known about their real effect when applied in women in China with FIGO stage IA-IIB cervical cancer. In addition, the incidence of specific complications associated with radical hysterectomy, and the influence of prognostic factors (such as uterine corpus invasion, and urologic complication) on tumor outcomes remain unclear. This large hospital-based cohort duly contributed several studies to answer above questions based on evidence in the database.

\section{Higher Risk of Major Surgical Complications With Laparoscopic Radical Hysterectomy (LRH) Compared With Abdominal Radical Hysterectomy (ARH)}

We compare a total of 18447 patients with FIGO stage IA-IIB cervical cancer diagnosed between 2004 and 2015 in China (34) to explore the rate of major complications associated with LRH (29.8\%) and ARH (70.2\%) for cervical cancer. We observed that the rate of LRH complications was $8.74 \%$ in 2009 and then was stabilized within the range of $4.80-6.56 \%$ between 2010 and 2015. The rate of ARH complications was relatively stable, with a range of $2.07-3.66 \%$. Notably, our study showed that the LRH group had significant higher odds of intraoperative complications $(\mathrm{OR}=3.88,95 \% \mathrm{CI} 2.47$ to 6.11$)$ and postoperative complications $(\mathrm{OR}=1.42,95 \% \mathrm{CI} 1.11$ to 1.82$)$ compared with the ARH group. Furthermore, compared with ARH, LRH was related to an increased risk of urologic complications, such as intraoperative ureteral injury, bowel injury, postoperative vesicovaginal fistula, ureterovaginal fistula, rectovaginal fistula, and chylous leakage. The results suggest that LRH for cervical cancer may not be as minimally invasive as it seems.

\section{Urologic Complications With Radical Hysterectomy for Cervical Cancer Seemed to Have an Implicit on Short-Term But Not on Long-Term Survival}

Of the 21026 patients undergoing radical hysterectomy for early-stage cervical cancer (35), we documented 324 (1.54\%) cases with at least one urologic complication. Variables significantly associated with a higher rate of urologic complications included being treated at a facility in a first-tier city (OR $=2.08,95 \%$ CI 1.24 to 3.48$)$, at a women's and children's hospital (OR $=2.26,95 \%$ CI 1.47 to 3.48$)$, and undergoing LRH $(\mathrm{OR}=4.68,95 \%$ CI 3.44 to 6.36$)$. Furthermore, our study demonstrated that the occurrence of urologic complications was an independent risk factor for decreased 2-year overall survival $(\mathrm{OS})(\mathrm{OR}=1.78,95 \% \mathrm{CI} 1.09$ to 2.92$)$, but not for decreased 5-year OS $(\mathrm{OR}=1.27,95 \%$ CI 0.83 to 1.94$)$. Additionally, age $\geq 60$, preoperative radiotherapy, FIGO stages IB2-IIB, positive pelvic lymph node, positive parametrial invasion, deep 1/2 stromal invasion, positive lymph vascular space (LVSI), tumor size $>4 \mathrm{~cm}$, and inadequate adjuvant treatment were also significant predictors of lower 5-year OS. 


\section{Compared With ARH, Robot-Assisted Radical Hysterectomy (RRH) or LRH for Cervical Cancer Predicted Worse Oncological Outcomes}

We published a study (36) that evaluated 3-year OS and diseasefree survival (DFS) of RRH and ARH for patients with cervical cancer whose FIGO stages were IA1 with LVSI-IIA2. After propensity score matching, patients who underwent RRH were likely to have shorter rates of 3-year OS $(94.4 \% v s$. $97.8 \%, \mathrm{p}=$ $0.002)$ and 3-year DFS $(91.1 \%$ vs. $95.4 \%, \mathrm{p}=0.001)$ than those who underwent $\mathrm{ARH}$. In multivariable models, $\mathrm{RRH}$ was associated with decreased rates of 3-year OS ( $\mathrm{HR}=2.86,95 \%$ CI 1.59 to 5.16 ) and 3-year DFS (HR $=2.34,95 \%$ CI 1.54 to 3.56 ). However, the ARH showed similar rates of 3-year oncological outcomes among patients with stage IB1 and tumor size $<2 \mathrm{~cm}$.

Another study (37) included 13413 participants with FIGO stage IA1 with LVSI-IIA2 cervical cancer. It showed that the rates of 5-year DFS of patients in the LRH group were lower than those in the ARH group ( $\mathrm{HR}=1.25,95 \%$ CI 1.11 to 1.40 ). Compared with ARH, LRH was not an appropriate approach for patients whose FIGO stage was IB1 or IIA1 and tumor size was $\geq 2 \mathrm{~cm}$. In other words, LRH or RRH could lead to worse oncological outcomes than ARH for patients with cervical cancer depending on the specific FIGO stage. These results demonstrate the need to be more cautious and considerate in the choice of surgical approaches.

\section{Uterine Corpus Invasion Should be Taken Seriously}

Diagnosis of uterine corpus invasion is frequently missed. According to a retrospective review of original pathology specimens (38) there were 38 patients with a missed diagnosis of uterine corpus invasion and 20 patients were misdiagnosed with uterine corpus invasion among 1414 patients with FIGO stage IA2-IIB cervical cancer from 11 medical institutions in mainland China. We found that myometrial invasion $\geq 50 \%$ seemed to be an independent prognostic factor for decreased rates of 5-year OS (HR $=2.74,95 \%$ CI 1.81 to 4.13 ) and 5-year DFS (HR $=2.31,95 \%$ CI 1.59 to 3.35 ), whereas myometrial invasion $<50 \%$ or endometrial invasion was not associated with outcomes of cervical cancer.

Between 2015 and 2020, the Four-C Study gave rise to 47 articles published in peer-reviewed journals. A list of publications will be available on the website currently under construction.

\section{FUTURE PLANS?}

We will further assess outcomes of different management strategies on cervical cancers with specific clinical stages. We will also continue to evaluate the influence of various prognostic factors on the oncological outcomes to guide treatment options, care, and follow-up.
Apart from that, we will also assess the natural history of cervical cancer, including biologic onset, subclinical stage, clinical stage, and outcome as much as possible, through a comprehensive analysis of the detailed information of patients who have not received any treatment or intervention. This will enable us to clarify the yield of different treatments compared with no treatment. The exploration of the natural history of cervical cancer will also contribute to promotion of the early diagnosis and prevention.

We are building a cervical cancer imaging database $(33,39)$ to collect the Dicom file data of computed tomography (CT)/ magnetic resonance imaging (MRI) examinations before treatment of cervical cancer. As of September 10, 2020, CT data of 3042 patients with cervical cancer and MRI data of 2843 patients with cervical cancer have been collected, among which 670 patients have both CT and MRI data. We have manually labeled the tumor boundaries and drawn the contours of the abdominal and pelvic lymph nodes on the collected original imaging. Moreover, we are collecting original pathological samples of patients with cervical cancer. Based on those, we may conduct extensive research on imaging omics and pathology omics of cervical cancer using $\mathrm{AI}$ and $\mathrm{ML}$ and advanced biological technology.

With digital medicine of obstetrics and gynecology, our team reconstructed a three-dimensional model of female abdominal and pelvic structure based on the computed tomography angiography (CTA) and MRI data sets of participants. We also constructed a digital diagnosis and treatment platform for gynecological and obstetrical diseases. More importantly, we creatively applied digital medicine technology to the preoperative diagnosis, intraoperative guidance, surgical navigation, digital delivery and postoperative evaluation of obstetrical and gynecological diseases.

Finally, our team has committed resources to diagnose difficult miscellaneous diseases using the three-dimensional modeling from the viewpoint of the source of arterial blood supply. In this clinical practice, a pelvic mass of an unknown source can also be successfully identified, which has improved the diagnosis rate of the pelvic mass of an unknown source.

\section{WHAT ARE THE MAIN STRENGTHS AND WEAKNESSES?}

This hospital-based cohort has several notable strengths. Foremost, it is one of the largest cervical cancer clinical databases to have been established and is likely to be the largest cervical cancer imaging and pathology database worldwide. A sufficiently large sample size allows us to carry out the study restricted to special-interest patient groups (e.g., patients with FIGO stage IB1 to IIA2 cervical cancer) with sufficient statistical power. And this resource may open the door to digital medical research on cervical cancer. Second, the cohort included not only cervical cancer patients who were treated with different measures, but also more than 1,400 cervical cancer patients who did not receive any treatment. It means that this cohort is able to provide 
us a unique opportunity to clarity the progression of cervical cancer and explore the real benefit of different therapeutic methods. Third, we have recruited participants in 26 cities of 14 provinces across the mainland China and created a network of 47 sites that include the different types and levels of hospitals. Of all patients (regardless of outpatients and inpatients) in attendance at the 47 sites were enrolled into the cohort. This makes it possible to roughly describe the burden of cervical cancer at different times and in different regions across mainland China, which could potentially inform prevention strategies. Forth, detailed information is available on socioeconomic characteristics, imaging, pathology, comorbidities, patient's management, complications, and other variables, enabling us to conduct comprehensive statistical analyses. Despite difficult working conditions, we have maintained inclusion and follow-up in this large cohort for over 6 years.

One weakness of this project is that some information on clinical outcomes prognoses relies on inpatient medical records, readmission records or outpatient records. As a result, some complications and other related events may be underreported and underestimated. In addition, although all laboratory staff strictly followed the procedure to perform each test, the results of laboratory tests may be affected by the equipment used in different hospitals, while it is really hard to get all 47 hospital to use the same equipment. Finally, several variables, such as those indicating economic status, lifestyle behaviors (smoking status, alcohol assumption and physical activity, etc.), living quality and utilization of medical resource, are not currently collected in the study because they may not be included in routine clinical practice and medical records, from which we obtained the information. We will endeavor to supplement those information during future follow-up, and we plan to include these variables when encountering a new patient with cervical cancer from July 2021.

\section{WHERE CAN I FIND OUT MORE? CAN I GET HOLD OF THE DATA?}

The databases remain the property of all participating centers and will still be managed by the Four-C Study group. Although the databases are not yet freely available in the public domain, potential collaborators are welcomed to contact the corresponding authors, Chun-Lin Chen (e-mail: ccl1@ smu.edu.cn) or Chen Mao (e-mail: maochen9@smu.edu.cn). Anyone can submit at least one specific study proposal describing the background, objectives and methods of the study. The databases can be partially transmitted to successful applicants with adequate statistical expertise. Otherwise, the members of the Four-C Study group will cooperatively analyze the data with the applicants. What's exciting is that we are trying our best to build a public website show the names of all variables and their detailed descriptions. By that moment, anyone may browse the website anytime and anywhere to learn about our project, and users interested in obtaining and using those data could also fill out a Data Use Agreement form downloading from the website to apply for access to the data.

\section{DATA AVAILABILITY STATEMENT}

The original contributions presented in the study are included in the article/supplementary material. Further inquiries can be directed to the corresponding authors.

\section{ETHICS STATEMENT}

The studies involving human participants were reviewed and approved by The institutional ethics committee of Nanfang Hospital. Written informed consent for participation was not required for this study in accordance with the national legislation and the institutional requirements.

\section{AUTHOR CONTRIBUTIONS}

Concept and design: C-LC, CM, J-HL, PL, X-RZ, Z-QL, and LXS. Data collection and cleaning: PL, P-FL, H-WZ, and B-LC, MJ, LW, SK, and on behalf of Chinese Cervical Cancer Clinical Study collaborative group. Statistical analysis, or interpretation of data: X-RZ, Z-QL, and Z-HL. Drafting of the manuscript: X$\mathrm{RZ}, \mathrm{Z}-\mathrm{QL}$, and L-XS. Critical revision of the manuscript for important intellectual content: C-LC, CM, J-HL, PL, H-WZ, and B-LC, MJ, LW, SK. Supervision: C-LC and CM. All authors contributed to the article and approved the submitted version.

\section{FUNDING}

This study was funded by the National Science and Technology Support Program of China (2014BAI05B03), the National Natural Science Fund of Guangdong (2015A030311024), the Science and Technology Plan of Guangzhou (158100075), Guangdong Medical Science and Technology Research Fund Project (A2020077), Basic and Applied Basic Research Fund of Guangdong Province (2019A1515110337), the Project Supported by Guangdong Province Universities and Colleges Pearl River Scholar Funded Scheme (2019), the Construction of High-level University of Guangdong (G820332010, G618339167 and G618339164), Chinese Postdoctoral Science Foundation (2019M660207), and Nanfang Hospital President Fund (2019C005). The funders played no role in the study design or implementation; data collection, management, analysis or interpretation; manuscript preparation, review or approval or the decision to submit the manuscript for publication.

\section{ACKNOWLEDGMENTS}

We are grateful for the providing the medical records by the network of 47 hospitals. We thank all members of Chinese Cervical Cancer Clinical Study collaborative group for assisting with the design and organization. Members of Chinese Cervical Cancer Clinical Study collaborative group: Li-Zhi Liang and 
Ji-Hong Liu, Sun Yat-sen University Cancer Center, Guangzhou, Guangdong, China; Zhu-Mei Cui, The Affiliated Hospital of Qingao University, Qingdao, Shandong, China; Yan Ni, Yuncheng Central Hospital, Yuncheng, Shanxi, China; Li-Hong Lin and $\mathrm{Yu}$ Guo, Anyang Cancer Hospital, Anyang, Henan, China; Wei-Dong Zhao, Anhui Provincial Cancer Hospital, HeFei, Anhui, China; Jian-Xin Guo, Daping Hospital, Army Medical University, Chonqing, Chongqing, China; Ying Yang, Xinqiao Hospital, Army Medical University, Chongqing, Chongqing, China; Wen-Tong Liang and Dong-Lin Li, Guizhou Provincial people's Hospital, Guiyang, Guizhou, China; Yi Zhang, The First Hospital of China Medical University, Shenyang, Liaoning, China; Wu-Liang Wang, The Second Affiliated Hospital of Zhengzhou University, Zhengzhou, Henan, China; Shao-Guang Wang, Yantai Yuhuangding Hospital, Yantai, Shandong, China; Dan-Bo Wang, Liaoning Cancer Hospital, Shenyang, Liaoning, China; Xue-Mei Zhan and Ming-Wei Li, Affiliated Jiangmen Hospital of Sun Yat-sen University, Jiangmen, Guangdong, China; Yong-Xiu Yang and Chang Liu, The First Hospital of Lanzhou University, Lanzhou, Gansu, China; Min Hao, The Second Hospital of Shanxi Medical University, Taiyuan, Shanxi, China; Wei-Feng Zhang, Ningbo Women \& Children's Hospital, Ningbo, Zhejiang, China; Pei-Yan Du, The Affiliated Cancer Hospital and Institute of Guangzhou Medical University, Guangzhou, Guangdong, China; Zi-Yu Fang, Liuzhou Workers' Hospital, Liuzhou, Guangxi, China; Rui Yang, Shenzhen Hospital of Peking University, Shenzhen, Guangdong, China; En-Cheng Dai and Rui-Lei Liu, Linyi People's Hospital, Linyi, Shandong, China; Long Chen, Qingdao Municipal Hospital, Qingdao, Shandong, China; Mu-Biao Liu and Yuan-Li He. Zhujiang Hospital, Southern Medical University, Guangzhou, Guangdong, China; Ji-Long Yao and Zhi-Hua Liu, Shenzhen Maternity \& Child Healthcare Hospital, Shenzhen, Guangdong, China; Qiu-Bo

\section{REFERENCES}

1. Bray F, Ferlay J, Soerjomataram I, Siegel RL, Torre LA, Jemal A. Global Cancer Statistics 2018: GLOBOCAN Estimates of Incidence and Mortality Worldwide for 36 Cancers in 185 Countries. CA Cancer J Clin (2018) 68:394424. doi: $10.3322 /$ caac. 21492

2. Fitzmaurice C, Akinyemiju TF, Al Lami FH, Alam T, Alizadeh-Navaei R, Allen C, et al. Global, Regional, and National Cancer Incidence, Mortality, Years of Life Lost, Years Lived With Disability, and Disability-Adjusted Life-Years for 29 Cancer Groups, 1990 to 2016: A Systematic Analysis for the Global Burden of Disease Study. JAMA Oncol (2018) 4:1553-68. doi: 10.1001/jamaoncol.2018.2706

3. Koh WJ, Greer BE, Abu-Rustum NR, Apte SM, Campos SM, Cho KR, et al. Cervical Cancer, Version 2.2015. J Natl Compr Canc Netw (2015) 13:395-404. doi: 10.6004/jnccn.2015.0055. quiz 404.

4. Ferlay J, Steliarova-Foucher E, Lortet-Tieulent J, Rosso S, Coebergh JW, Comber $\mathrm{H}$, et al. Cancer Incidence and Mortality Patterns in Europe: Estimates for 40 Countries in 2012. Eur J Cancer (2013) 49:1374-403. doi: 10.1016/j.ejca.2012.12.027

5. Abu-Rustum NR, Yashar CM, Bean S, Bradley K, Campos SM, Chon HS, et al. Nccn Guidelines Insights: Cervical Cancer, Version 1.2020. J Natl Compr Canc Netw (2020) 18:660-6. doi: 10.6004/jnccn.2020.0027

6. Wild CP, Weiderpass E, Stewart BW. World Cancer Report: Cancer Research for Cancer Prevention. Lyon, France: International Agency for Research on Cancer. (2020). Available at: http://publications.iarc.fr/586.

7. Hall MT, Simms KT, Lew JB, Smith MA, Brotherton JM, Saville M, et al. The Projected Timeframe Until Cervical Cancer Elimination in Australia: A
Lv, Beijing Hospital, Beijing, Beijing, China; Yuan-Guang Meng, The 7th Medical Center, Chinese PLA General Hospital, Beijing, Beijing, China; An-Wei Lu, The Maternity and Child Care Hospital of Guizhou Province, Guiyang, Guizhou, China; Shuang-Ling Jin, Peace Hospital affiliated to Changzhi Medical College, Changzhi, Shanxi, China; Qing-Huang Xie, Foshan Women and Children Hospital, Foshan, Guangdong, China; Yan Xu, Guangzhou Panyu Central Hospital, Guangzhou, Guangdong, China; Hai-Lin Wang, Gansu Provincial Hospital, Lanzhou, Gansu, China; Ben Ma, Guangzhou First People's Hospital, Guangzhou, Guangdong, China; Zhong-Hai Wang, Huazhong University of Science and Technology Union Shenzhen Hospital (Nanshan Hospital), Shenzhen, Guangdong, China; Lin Zhu, The Second Hospital of Shandong University, Jinan, Shandong, China; Bin Ling, China-Japan Friendship Hospital, Beijing, Beijing, China; Qian-Yong Zhu, The No.153 Central Hospital of PLA, Pingdingshan, Henan, China; XiaoHong Wang, Department of Gynecology, Laiwu People's Hospital, Jinan, Shandong, China; Ding-Yuan Zeng and Zhong Lin, Maternal and Child Health Care Hospital of Liuzhou, Liuzhou, Guangxi, China; Bin Zhu, Yiwu Branch of Children's Hospital Zhejiang University School of Medicine (Yiwu Maternity and Children Hospital), Yiwu, Zhejiang, China; Jun-Shen He, Nanfang Hospital, Southern Medical University, Guangzhou, Guangdong, China; Xiao-Lin Chen, Nanfang Hospital, Southern Medical University, Guangzhou, Guangdong, China; Hui-Jian Fan, Nanfang Hospital, Southern Medical University, Guangzhou, Guangdong, China; Lu Wang, Nanfang Hospital, Southern Medical University, Guangzhou, Guangdong, China; Jia-Ming Chen, Nanfang Hospital, Southern Medical University, Guangzhou, Guangdong, China; Lu Yin, Nanfang Hospital, Southern Medical University, Guangzhou, Guangdong, China. for providing medical records.

Modelling Study. Lancet Public Health (2019) 4:e19-27. doi: 10.1016/s2468 2667(18)30183-x

8. WHO. Who Director-General Calls for All Countries to Take Action to Help End the Suffering Caused by Cervical Cancer (2018). Available at: http://www. who.int/reproductivehealth/call-to-action-elimination cervical-cancer/en/ (Accessed May 22, 2018). Updated May 19.

9. International Papillomavirus Society. IPVS Statement: Moving Towards Elimination of Cervical Cancer as a Public Health Problem (2018). Available at: http://ipvsoc.org/wp-content/uploads/2018/02/IPVs statement-onelimination.pdf (Accessed May 2, 2018).

10. Youn JW, Hur SY, Woo JW, Kim YM, Lim MC, Park SY, et al Pembrolizumab Plus GX-188E Therapeutic DNA Vaccine in Patients With HPV-16-positive or HPV-18-Positive Advanced Cervical Cancer: Interim Results of a Single-Arm, Phase 2 Trial. Lancet Oncol (2020) 21:1653-60. doi: 10.1016/s1470-2045(20)30486-1

11. Kamath SD, Kircher SM, Benson AB. Comparison of Cancer Burden and Nonprofit Organization Funding Reveals Disparities in Funding Across Cancer Types. J Natl Compr Canc Netw (2019) 17:849-54. doi: 10.6004/jnccn.2018.7280

12. Matsuo K, Mandelbaum RS, Klar M, Ciesielski KM, Matsushima K, Matsuzaki $\mathrm{S}$, et al. Decreasing Utilization of Minimally Invasive Hysterectomy for Cervical Cancer in the United States. Gynecol Oncol (2021) S0090-8258(21) 00364-4. doi: 10.1016/j.ygyno.2021.05.005

13. Murakami N, Asami Y, Yoshida H, Takayanagi D, Hirose S, Kuno I, et al. Distribution of Genetic Alterations in High-Risk Early-Stage Cervical Cancer Patients Treated With Postoperative Radiation Therapy. Sci Rep (2021) 11:10567. doi: 10.1038/s41598-021-90139-0 
14. Shamseddine AA, Burman B, Lee NY, Zamarin D, Riaz N. Tumor Immunity and Immunotherapy for HPV-Related Cancers. Cancer Discov (2021). doi: 10.1158/2159-8290.Cd-20-1760

15. Armbrust R, Chen F, Richter R, Muallem MZ, Mustea A, Holthaus B, et al. Results of a German Wide Survey Towards Current Surgical Approach in Early Stage Cervical Cancer NOGGO Monitor 11. Sci Rep (2021) 11:9774. doi: 10.1038/s41598-021-89071-0

16. Zhang J, Cao D, Yang J, Shen K, He Y, Xue H. Tumor Volume Predicts HighRisk Patients and Guides Initial Chemoradiotherapy for Early Cervical Cancer. Front Oncol (2021) 11:640846. doi: 10.3389/fonc.2021.640846

17. Yang H, Chen M, Mei Z, Xie C, Zhou Y, Qiu H. Effectiveness and Prognostic Factors of Apatinib Treatment in Patients With Recurrent or Advanced Cervical Carcinoma: A Retrospective Study. Cancer Med (2021). doi: $10.1002 /$ cam4.3966

18. Mondini M, Deutsch E. (Chemo)Radiotherapy-Immunotherapy Combinations: Time to Get Tailored? Clin Cancer Res (2021) clincanres.1173.2021. doi: 10.1158/1078-0432.Ccr-21-1173

19. Chen C, Cheng X, Li S, Chen H, Cui M, Bian L, et al. A Novel Signature for Predicting Prognosis of Smoking-Related Squamous Cell Carcinoma. Front Genet (2021) 12:666371. doi: 10.3389/fgene.2021.666371

20. Zhang J, Sun M, Li N, Miao M, Yang Y, Hsu HC, et al. Contemporary External Beam Radiotherapy Boost or High Dose-Rate Brachytherapy Boost for Cervical Cancer: A Propensity-Score-Matched, Nationwide, Population-Based Cohort Study. Am J Cancer Res (2021) 11:1719-32. doi: 10.2139/ssrn.3727317

21. Xu M, Wu Q, Cai L, Sun X, Xie X, Sun P. Systemic Inflammatory Score Predicts Overall Survival in Patients With Cervical Cancer. J Cancer (2021) 12:3671-7. doi: 10.7150/jca.56170

22. Yan W, Jin H, Zhang X, Long S, Xia Q, Meng D, et al. Identification of an Immune Signature to Predict Poor Clinical Outcome in Cervical Cancer. Epigenomics (2021) 13:891-907. doi: 10.2217/epi-2020-0437

23. Xue P, Ng MTA, Qiao Y. The Challenges of Colposcopy for Cervical Cancer Screening in LMICs and Solutions by Artificial Intelligence. BMC Med (2020) 18:169. doi: 10.1186/s12916-020-01613-x

24. Xue P, Tang C, Li Q, Li Y, Shen Y, Zhao Y, et al. Development and Validation of an Artificial Intelligence System for Grading Colposcopic Impressions and Guiding Biopsies. BMC Med (2020) 18:406. doi: 10.1186/s12916-020-01860-y

25. Dueñas-González A, Cetina L, Coronel J, Martínez-Baños D. Pharmacotherapy Options for Locally Advanced and Advanced Cervical Cancer. Drugs (2010) 70:403-32. doi: 10.2165/11534370-000000000-00000

26. Barbera L, Thomas G. Management of Early and Locally Advanced Cervical Cancer. Semin Oncol (2009) 36:155-69. doi: 10.1053/j.seminoncol.2008.12.007

27. Rose PG. Combined-Modality Therapy of Locally Advanced Cervical Cancer. J Clin Oncol (2003) 21:211s-7s. doi: 10.1200/jco.2003.01.222

28. Iqbal MJ, Javed Z, Sadia H, Qureshi IA, Irshad A, Ahmed R, et al. Clinical Applications of Artificial Intelligence and Machine Learning in Cancer Diagnosis: Looking Into the Future. Cancer Cell Int (2021) 21:270. doi: 10.1186/s12935-021-01981-1

29. Wiens J, Shenoy ES. Machine Learning for Healthcare: On the Verge of a Major Shift in Healthcare Epidemiology. Clin Infect Dis (2018) 66:149-53. doi: $10.1093 / \mathrm{cid} / \mathrm{cix} 731$
30. Landhuis E. Deep Learning Takes on Tumours. Nature (2020) 580:551-3. doi: 10.1038/d41586-020-01128-8

31. McLauchlan J, Innes H, Dillon JF, Foster G, Holtham E, McDonald S, et al. Cohort Profile: The Hepatitis C Virus (HCV) Research Uk Clinical Database and Biobank. Int J Epidemiol (2017) 46:1391-1391h. doi: 10.1093/ije/dyw362

32. Liu P, Lin L, Kong Y, Huo Z, Zhu L, Bin X, et al. Comparison of Survival Outcomes Between Radio-Chemotherapy and Radical Hysterectomy With Postoperative Standard Therapy in Patients With Stage IB1 to IIA2 Cervical Cancer: Long-Term Oncological Outcome Analysis in 37 Chinese Hospitals. BMC Cancer (2020) 20:189. doi: 10.1186/s12885-020-6651-8

33. Chen C, Liu P, Ni Y, Tang L, Xu Y, Bin X, et al. Laparoscopic Versus Abdominal Radical Hysterectomy for Stage IB1 Cervical Cancer Patients With Tumor Size $\leq 2$ Cm: A Case-Matched Control Study. Int J Clin Oncol (2020) 25:937-47. doi: 10.1007/s10147-020-01630-Z

34. Liang C, Liu P, Cui Z, Liang Z, Bin X, Lang J, et al. Effect of Laparoscopic Versus Abdominal Radical Hysterectomy on Major Surgical Complications in Women With Stage IA-IIB Cervical Cancer in China, 2004-2015. Gynecol Oncol (2020) 156:115-23. doi: 10.1016/j.ygyno.2019.10.032

35. Liu P, Liang C, Lu A, Chen X, Liang W, Li D, et al. Risk Factors and LongTerm Impact of Urologic Complications During Radical Hysterectomy for Cervical Cancer in China, 2004-2016. Gynecol Oncol (2020) 158:294-302. doi: 10.1016/j.ygyno.2020.05.029

36. Chen B, Ji M, Li P, Liu P, Zou W, Zhao Z, et al. Comparison Between RobotAssisted Radical Hysterectomy and Abdominal Radical Hysterectomy for Cervical Cancer: A Multicentre Retrospective Study. Gynecol Oncol (2020) 157:429-36. doi: 10.1016/j.ygyno.2020.02.019

37. Li P, Liu P, Yang Y, Wang L, Liu J, Bin X, et al. Hazard Ratio Analysis of Laparoscopic Radical Hysterectomy for IA1 With LVSI-IIA2 Cervical Cancer: Identifying the Possible Contraindications of Laparoscopic Surgery for Cervical Cancer. Front Oncol (2020) 10:1002. doi: 10.3389/fonc.2020.01002

38. He F, Li W, Liu P, Kang S, Sun L, Zhao H, et al. Influence of Uterine Corpus Invasion on Prognosis in Stage IA2-IIB Cervical Cancer: A Multicenter Retrospective Cohort Study. Gynecol Oncol (2020) 158:273-81. doi: 10.1016/j.ygyno.2020.05.005

39. Tian X, Sun C, Liu Z, Li W, Duan H, Wang L, et al. Prediction of Response to Preoperative Neoadjuvant Chemotherapy in Locally Advanced Cervical Cancer Using Multicenter Ct-Based Radiomic Analysis. Front Oncol (2020) 10:77. doi: $10.3389 /$ fonc. 2020.00077

Conflict of Interest: The authors declare that the research was conducted in the absence of any commercial or financial relationships that could be construed as a potential conflict of interest.

Copyright (c) 2021 Zhang, Li, Sun, Liu, Li, Li, Zhao, Chen, Ji, Wang, Kang, Lang, Mao and Chen. This is an open-access article distributed under the terms of the Creative Commons Attribution License (CC BY). The use, distribution or reproduction in other forums is permitted, provided the original author(s) and the copyright owner(s) are credited and that the original publication in this journal is cited, in accordance with accepted academic practice. No use, distribution or reproduction is permitted which does not comply with these terms. 